\title{
ALTERNATIVAS TERAPÉUTICAS EN EL CÁNCER DE PRÓSTATA LOCALIZADO. EXPERIENCIA SOBRE 454 PACIENTES
}

\author{
M. ARRIZABALAGA MORENO, J.I. GARCÍA GONZÁLEZ, M.J. PÉREZ GARNELO, \\ P. PANIAGUA ANDRÉS
}

Servicio de Urología. Hospital de Móstoles. Móstoles (Madrid).

Actas Urol Esp. 28 (6): 418-431, 2004

\section{RESUMEN}

ALTERNATIVAS TERAPÉUTICAS EN EL CÁNCER DE PRÓSTATA LOCALIZADO. EXPERIENCIA SOBRE 454 PACIENTES

OBJETIVOS: Evaluar en una cohorte de 454 pacientes con cáncer de próstata no diseminado tratados con diversas alternativas terapéuticas la supervivencia libre de progresión (SLP), supervivencia global (SG) y supervivencia específica (SE) en función de distintos factores pronósticos y comparar nuestros resultados con la bibliografia.

MATERIAL Y MÉTODOS: Entre 1983 y 2000 hemos diagnosticado 706 pacientes de cáncer de próstata, de los que 454 eran clínicamente tumores no diseminados. Los tratamientos utilizados para estos 454 pacientes han sido: observación (OBS) (103 pacientes), prostatectomía radical (PR) (108), radioterapia no asociada a tratamiento hormonal (RT) (148) y bloqueo hormonal (BH) (95). Hemos analizado la SLP, SG y SE en cada grupo y comparativamente en función de distintos factores pronósticos en el momento del diagnóstico: edad, PSA, Gleason y estadio. También analizamos la repercusión de la progresión en la SG. El seguimiento medio ha sido de 5,6 años (0,1-19,2 años; mediana 5,2).

RESULTADOS: SLP: han progresado 145 pacientes (32\%), a 5 años la SLP para OBS: 77\%, PR: 61\%, RT: 63\%, BH: $73 \%$. A 10 años: 67 , 50, 25 y 67\%, respectivamente. No diferencias significativas entre PR y RT. En pacientes con PSA $<10$ y Gleason $<8$ no diferencias entre OBS, PR y RT. SG: han fallecido 126 pacientes (28\%), a 5 años la SG fue: 80, 90, 85 y $64 \%$ y a 10 años: 61, 76, 67 y $32 \%$. No diferencias entre OBS, PR y RT. SE: han fallecido por su tumor 31 (6,8\%). SE a 5 años: 100, 98, 97 y 83\%. A 10 años: 94, 98, 88 y $77 \%$. No diferencias en la SG entre los pacientes en progresión comparados con los pacientes sin progresión tumoral en los tratados con OBS, PR y RT.

CONCLUSIONES: La determinación del antígeno PSA ha trasladado el diagnóstico del cáncer de próstata a estadios muy precoces, sin embargo nuestros datos y la revisión de la bibliografia no permiten definir cual es la mejor estrategia terapéutica incluyendo la alternativa observacional. Debemos dar la suficiente información individualizada tanto en la fase de diagnóstico precoz como a la hora de decidir un tratamiento.

PALABRAS CLAVE: Cáncer de próstata. Prostatectomía radical. Observación. Tratamiento radioterápico. Supervivencia. Factores pronósticos.

\section{ABSTRACT}

THERAPEUTIC OPTIONS FOR PATIENTS UIT LOCALIZED PROSTATIC CARCINOMA: OUR EXPERIENCE WITH 454 PATIENTS

OBJECTIVES: To evaluate the influence of different therapeutic options on progression-free survival (PFS), overall survival (OS) and specific survival (SS) in a cohort of 454 patients with localized prostatic carcinoma, taking into account different prognostic factors, and to compare our results to those reported in the world literature.

MATERIAL AND METHODS: Between 1983 and 2000 we have diagnosed 706 new cases of prostatic carcinoma and 454 were clinically localized tumors. The different therapeutic options employed in our series of patients have been: follow-up (FU) (103 patients); radical prostatectomy (RP) (108 patients); radiotherapy without hormonal blockade (RT) (148 patients); and hormonal blockade (HB) (95 patients). We have determined the PFS, the OS and the SS for each group of patients and compared them in patients with different prognostic factors at the time of diagnosis, including age, PSA levels, Gleason's grading and TNM staging. We have also analysed the influence of the tumor progression on the OS. The mean follow-up time has been 5.6 years (range: 0.1-19.2; median: 5.2).

RESULTS: For PFS: the disease progressed in 145 patients (32\%) and the PFS at 5 and 10 years has been $77 \%$ and $67 \%$ for FU; $61 \%$ and $50 \%$ for RP; $63 \%$ and $25 \%$ for RT; and $73 \%$ and $67 \%$ for HB, respectively. The differences between RT and RP were not statistically significant. For the subgroup of patients with PSA levels $<10$ and Gleason $<8$ the differences between FU, RP and RT did not reach statistical significance. For OS: 126 patients of our series died (28\%) and the OS at 5 and 10 years has been $80 \%$ and $61 \%$ for FU; $90 \%$ and $76 \%$ for RP; $85 \%$ and $67 \%$ for RT; and $64 \%$ and $32 \%$ for HB, respectively. We have found no significant differences between FU, RP and RT. For SS: 31 patients of our series died of disease (6.8\%). The SS at 5 and 10 years has been $100 \%$ and $94 \%$ for FU; $98 \%$ and $98 \%$ for RP; $97 \%$ and $88 \%$ for RT; and $83 \%$ and $77 \%$ for HB, respectively. We have found no significant differences in the OS between patients with disease progression and without disease progression treated with FU, RP and RT.

CONCLUSIONS: Determination of PSA levels has allowed diagnosis of prostatic carcinomas in early stages of disease; however, our results and those reported in the literature cannot define which is the best therapeutic option in these patients. We should offer the patients individualized information both in the phase of early diagnosis and of therapeutic decisions.

KEY WORDS: Prostatic carcinoma. Radical prostatectomy. Watchful waiting. Radiotherapy. Survival. Prognostic factors. 
$\mathrm{E}^{1}$ cáncer de próstata es el tumor urológico más frecuente en el varón. Su detección cada vez más precoz está aumentando, fundamentalmente, por la utilización del antígeno prostático específico (PSA). Las determinaciones de este marcador tumoral se realizan tanto en las consultas urológicas como en medicina primaria o en exámenes de empresa. Esta situación ha producido cambios importantes tanto en la incidencia como en el manejo de esta enfermedad en los últimos 20 años. Esta incidencia para la Comunidad de Madrid fue de 100 pacientes por 100.000 varones en el año $2000^{1}$, cifra que triplica otra publicada en 1997 para la misma zona sanitaria ${ }^{2}$.

Sin embargo, persisten las incógnitas sobre cual es el mejor manejo de esta enfermedad en sus distintas fases. En fase de tumor localizado las alternativas terapéuticas en la actualidad son múltiples: observación, prostatectomía radical, radioterapia externa, braquiterapia, HIFU, crioterapia, antiandrógenos, bloqueo hormonal máximo, etc. En la actualidad recientes publicaciones cuestionan el potencial beneficio del tratamiento del cáncer de próstata diagnosticado precozmente ${ }^{3,4}$.

En el presente trabajo analizamos nuestros resultados obtenidos en relación con la supervivencia libre de progresión, supervivencia global y supervivencia específica en 454 pacientes con cáncer de próstata no diseminado al diagnóstico tratados con distintas alternativas terapéuticas y comparamos estos resultados con la literatura.

\section{MATERIAL Y MÉTODOS}

Entre el año 1983 y el año 2000 se han diagnosticado en nuestro Servicio 706 pacientes de adenocarcinoma de próstata en los que existe comprobación anatomopatológica. En 454 pacientes $(64,3 \%)$ se objetivaron tumores localizados o localmente avanzados, siendo este grupo el objetivo central del trabajo.

PROTOCOLO DE DIAGNÓSTICO, ESTADIAJE, TRATAMIENTO Y SEGUIMIENTO

Nuestro protocolo para el diagnóstico, estudio de extensión y tratamiento del cáncer de próstata ha ido evolucionando a lo largo del periodo de estudio. El diagnóstico histológico se ha realizado mediante tres métodos: biopsia transrrectal, resección transuretral y adenomectomía suprapúbica. Hemos utilizado el método de Gleason para la gradación anatomopatológica, habiéndose podido establecer en 427 pacientes $(94,1 \%)$.

El estadiaje clínico se ha hecho basándonos en el tacto rectal, en la ecografía transrrectal así como en las biopsias del diagnóstico. Para el estudio de extensión hemos utilizado la radiografía de tórax y hasta el año 1996 en todos los pacientes: CT abdominal, gammagrafía ósea y otras exploraciones si hubieran sido necesarias. Desde el año 1989 disponemos de determinación de PSA, el cual se ha podido realizar previo al diagnóstico en 391 pacientes (86\%). Desde el año 1997 a raíz de un estudio en el que evaluamos la sensibilidad y especificidad del PSA para detectar metástasis del tumor en nuestra serie, sólo realizamos gammagrafia ósea en pacientes con un PSA al diagnóstico superior a $10 \mathrm{ng} / \mathrm{ml}$ o un Gleason superior a 7 .

De los 454 pacientes tienen linfadenectomía 118 que corresponden a 108 pacientes tratados con prostatectomía radical y 10 pacientes programados para cirugía radical en los que la biopsia intraoperatoria reveló afectación ganglionar y se suspendió la cirugía. No tienen linfadenectomía 336 pacientes. El resultado de la linfadenectomía no se ha utilizado para el estadiaje clínico al diagnóstico.

Se ha utilizado la clasificación TNM de 1997. Hemos considerado tumores localizados a los tumores T1-2, NO, MO. Tumores localmente avanzados han sido: T3-4, NO-X, MO. Tumores diseminados: cualquier $\mathrm{T}$ asociado a $\mathrm{N}+\mathrm{y} / \mathrm{o}$ $\mathrm{M}+$. Los pacientes con PSA al diagnóstico superior a 100, independiente del resultado del estudio de extensión, se han considerado como diseminados.

Las alternativas terapéuticas que hemos utilizado para tumores no diseminados han sido cuatro: observación (103 pacientes), prostatectomía radical (108 pacientes), radioterapia (148 pacientes) y tratamiento hormonal (95 pacientes). La técnica que hemos utilizado de prostatectomía radical ha sido la retropúbica de Walsh asociada a linfadenectomía. En los pacientes tratados con radioterapia ésta ha sido externa y sólo se han seleccionado aquellos enfermos en los que el tra- 
tamiento ha sido de intención radical y no se ha asociado a tratamientos hormonales neoadyuvantes, concomitantes o adyuvantes.

Entre los 95 enfermos tratados con bloqueo hormonal están los 10 pacientes con tumores localizados al diagnóstico y con linfadenectomía positiva en los que se suspendió la cirugía radical y fueron tratados con supresión hormonal. En total de estos pacientes realizamos bloqueo androgénico máximo con un agonista LH RH asociado a un antiandrógeno en $89(93,7 \%)$, los otros $6(6,3 \%)$ fueron tratados con castración quirúrgica asociado a un antiandrógeno.

En 3 de los 108 pacientes tratados con prostatectomía radical hemos encontrado ganglios positivos (N1) en el estudio en formol, no detectados en la biopsia intraoperatoria. Estos enfermos se trataron posteriormente con observación.

La decisión del tratamiento la ha realizado el paciente, (o los familiares en el caso de pacientes ancianos), aconsejados por nosotros $\mathrm{y}$ basándonos en tres pilares fundamentales: clínica, carga tumoral y edad del enfermo. En pacientes menores de 70 años hemos utilizado sobre todo cirugía radical, radioterapia y observación. En pacientes mayores de 70 años: radioterapia, bloqueo hormonal y observación. Desde 1999 no hemos utilizado el tratamiento hormonal en estos estadios salvo por razones de sintomatología clínica.

Una vez realizado el tratamiento los pacientes han seguido revisiones periódicas que incluyen tacto rectal y determinación de PSA, así como otras exploraciones si hubieran sido necesarias. En los dos primeros años estas revisiones han tenido frecuencia trimestral, desde el segundo al quinto año semestral y desde el quinto año anual.

\section{CRITERIOS DE PROGRESIÓN}

Hasta que dispusimos de PSA en 1989 la progresión local se estableció por biopsia y la diseminación por pruebas clínicas (CT, gammagrafia ósea, etc.). Desde que disponemos de PSA, éste ha sido el factor fundamental de control de la enfermedad asociado a otras pruebas si ha sido preciso.

En los pacientes en los que se ha realizado prostatectomía radical se ha considerado progre- sión al incremento del PSA por encima de 0,5 $\mathrm{ng} / \mathrm{ml}$. En los pacientes sometidos a radioterapia la progresión se ha establecido con cualquier incremento sostenido del PSA por encima del nadir. En los pacientes sometidos a observación o a tratamiento hormonal la progresión se ha definido como el incremento sostenido del nivel de PSA por encima del nadir.

En todos los casos de progresión se han realizado tres determinaciones seriadas de PSA. Cuando estas determinaciones han confirmado la progresión la fecha que hemos utilizado como inicio de la misma es la primera determinación. No hemos tenido ningún caso de progresión clínica en la que no haya existido progresión bioquímica.

Los pacientes sometidos a prostatectomía radical o radioterapia hasta 1998 que han entrado en progresión se les ha realizado tratamiento de supresión hormonal. Desde 1998 los nuevos enfermos de este grupo que han entrado en progresión sin sintomatología clínica se les ha dejado en observación sin ningún tratamiento.

Los pacientes sometidos a observación que han entrado en progresión se han tratado de distintas formas: prostatectomía radical, radioterapia, bloqueo hormonal y mantenerse en observación si no tienen clínica.

\section{SEGUIMIENTO}

El periodo de inclusión de enfermos comienza en 1983 y termina en diciembre de 2000. Los historiales de los pacientes no fallecidos están actualizados al periodo 2001-2003, a excepción de 9 enfermos (2\%) perdidos para seguimiento con anterioridad al año 2001. Han fallecido 126 pacientes $(27,8 \%)$. Permanecen vivos al final del estudio $328(72,2 \%)$.

$\mathrm{El}$ seguimiento medio para los no fallecidos es de 6,1 años (IC al 95\% para la media: 5,8-6,5 años; mínimo 1,1-máximo 16,4 años; mediana: 5,8 años). Seguimiento medio de los 9 pacientes perdidos: 4,9 años (IC: 1,8-7,9; min: 1,1-máx: 12,9 años; mediana: 3,7 años). Seguimiento medio para fallecidos: 4,3 años (IC: 3,8-4,9 años; min: 0,1-máx: 19,5 años; mediana: 3,8 años). Seguimiento medio total de toda la muestra: 5,6 años (IC: 5,3-5,9 años; min: 0,1-máx: 19,5 años; mediana: 5,2 años). 


\section{OBJETIVOS}

El objetivo fundamental del trabajo es evaluar la supervivencia libre de progresión (SLP), supervivencia global (SG) y supervivencia específica o tumor dependiente (SE) en cada uno de los distintos grupos terapéuticos del estudio. Los criterios básicos de comparación han sido los datos obtenidos en el momento del diagnóstico clínico y no los obtenidos tras la prostatectomía radical en aquellos pacientes en los que se hubiera realizado esta opción terapéutica. Los factores analizados han sido: edad, PSA, Gleason y estadio al diagnóstico. Analizamos la evolución de los distintos grupos a lo largo del periodo de estudio tanto como variable continua como agrupados en periodos temporales.

Hemos agrupado estos distintos factores en dos grupos fundamentales. Grupo de pacientes con factores de teórico buen pronóstico: pacientes con PSA al diagnóstico menor de 10, Gleason menor de 7 y estadio localizado (T1-2). Grupo de pacientes con factores de teórico mal pronóstico en base al PSA (mayor o igual a 10) $\mathrm{y}$ al Gleason del diagnóstico (Gleason 8, 9 ó 10). Otro grupo de mal pronóstico se ha realizado con el corte del PSA en igual o mayor a $20 \mathrm{ng} / \mathrm{ml}$.

Otro resultado que hemos analizado es valorar la influencia que haya podido tener la progresión inicial en la supervivencia global.

\section{ANÁLISIS ESTADÍSTICO}

Para comparar variables cualitativas entre los distintos grupos se ha utilizado la prueba del $\mathrm{Chi}^{2}$, para variables cuantitativas hemos utilizado la prueba de la $\mathrm{t}$ de Student y el ANOVA con la prueba de Bonferroni para el análisis entre distintas variables. El análisis de la supervivencia se ha realizado por el método actuarial de Kaplan y Meier. La comparación entre los distintos grupos se ha hecho en análisis univariante mediante la prueba log-rank. En aquellos factores en los que ha existido significación estadística en análisis univariante se ha realizado análisis multivariante mediante regresión de Cox. Las pruebas de contraste de hipótesis se han realizado a dos colas. El nivel de significación se ha establecido en el 95\% $(\mathrm{p}<0,05)$.

\section{RESULTADOS}

\section{CARACTERISTTICAS DE LOS PACIENTES}

En la Tabla 1 se reflejan las características de los pacientes en cada grupo. Los enfermos tratados con prostatectomía radical tienen la edad media más baja y los tratados con bloqueo hormonal la mayor edad. Las diferencias en la edad media son estadísticamente significativas entre todos los grupos $(\mathrm{p}=0,00)$ a excepción de los tratados con radioterapia y los sometidos a observación ( $\mathrm{p}=\mathrm{NS}$ ). Las diferencias en el PSA medio de los tratados con observación, prostatectomía y radioterapia no son significativas. El PSA medio superior de los tratados con bloqueo hormonal sí es estadísticamente significativo con el resto de los grupos $(\mathrm{p}=0,00)$.

La distribución de los distintos estadios en los grupos no es homogénea habiendo diferencias estadísticamente significativas $(p=0,00)$. En los pacientes sometidos a observación sobre todo hay tumores $\mathrm{T} 1$. Al analizar los subgrupos del estadio T1 sólo hay diferencias con significación estadística entre los pacientes tratados con observación y el resto $(\mathrm{p}=0,00)$, en este grupo hay sobre todo tumores Tla y en el resto sobre todo tumores Tlc. Los tratados con prostatectomía radical y con radioterapia tienen una distribución en estadios homogénea sin diferencias estadísticamente significativas. Los tratados con bloqueo hormonal son pacientes con estadios sobre todo T2 y T3.

La distribución del Gleason entre los tratados con $\mathrm{PR}$ y radioterapia es homogénea $(\mathrm{p}=\mathrm{NS})$. El grupo de pacientes en observación está integrado sobre todo por Gleason bajo con diferencias significativas con el resto de grupos $(\mathrm{p}=0,00)$. Los tratados con bloqueo hormonal están integrados por Gleason más elevados que en el resto de grupos con significación estadística $(\mathrm{p}=0,00)$.

Al evaluar los grupos en función del número de pacientes incluidos en cada factor pronóstico analizado, no ha habido diferencias estadísticamente significativas entre los tratados con prostatectomía radical y los tratados con radioterapia. Sí ha habido diferencias entre los pacientes sometidos a observación integrados sobre todo por pacientes con factores pronósticos favorables en relación con el resto $(\mathrm{p}=0,01)$. También ha habido diferencias entre los tratados con bloqueo 
Tabla 1. Características de los pacientes en cada grupo de tratamiento

\begin{tabular}{|c|c|c|c|c|c|}
\hline & Observación & Prostatectomia & Radioterapia & $\begin{array}{l}\text { Bloqueo } \\
\text { hormonal }\end{array}$ & Todos \\
\hline & & & № (\%) & & \\
\hline No pacientes & $103(22,7)$ & $108(23,8)$ & $148(32,6)$ & $95(20,9)$ & $454(100)$ \\
\hline Edad media & 71,5 & 62,7 & 70,6 & 76,6 & 70,2 \\
\hline $\begin{array}{l}\text { PSA medio } \\
(\mathrm{ng} / \mathrm{ml})\end{array}$ & 10,3 & 11,1 & 13,8 & 30,6 & 15,9 \\
\hline \multicolumn{6}{|c|}{ Métodos diagnósticos } \\
\hline RTU & $52(50,5)$ & $9(8,3)$ & $41(27,7)$ & $21(22,1)$ & $123(27,1)$ \\
\hline Adenomectomía & $17(16,5)$ & 0 & $1(0,7)$ & 0 & $18(4)$ \\
\hline Biopsia TR & 34 (33) & $99(91,6)$ & $106(71,6)$ & $74(77,9)$ & $313(68,9)$ \\
\hline \multicolumn{6}{|l|}{ Estadios } \\
\hline $\mathrm{Tx}$ & $2(1,9)$ & 0 & 0 & $5(5,3)$ & $7(1,5)$ \\
\hline $\mathrm{T} 1$ & $77(74,8)$ & $34(31,5)$ & 37 (25) & $9(9,5)$ & $157(34,6)$ \\
\hline Tla & $45(58,4)$ & $1(2,9)$ & $4(10,8)$ & $1(11,1)$ & $53(33,8)$ \\
\hline $\mathrm{T} 1 \mathrm{~b}$ & $8(10,4)$ & $3(8,8)$ & $4(10,8)$ & $1(11,1)$ & $14(8,9)$ \\
\hline Tlc & $21(27,3)$ & $29(85,3)$ & $25(67,6)$ & $7(77,8)$ & $82(52,2)$ \\
\hline Desconocido & $3(3,9)$ & $1(2,9)$ & $4(10,8)$ & 0 & $8(5,1)$ \\
\hline $\mathrm{T} 2$ & $23(22,3)$ & $70(64,8)$ & $102(68,9)$ & $52(54,7)$ & $247(54,4)$ \\
\hline $\mathrm{T} 3$ & $1(1)$ & $4(3,7)$ & $8(5,4)$ & $21(22,1)$ & $34(7,5)$ \\
\hline $\mathrm{T} 4$ & 0 & 0 & $1(0,7)$ & $8(8,4)$ & $9(2)$ \\
\hline \multicolumn{6}{|l|}{ Gleason } \\
\hline $2-4$ & $64(62,1)$ & $22(20,4)$ & $39(26,4)$ & $12(12,7)$ & $137(30,2)$ \\
\hline $5-7$ & $31(30,1)$ & $66(61,1)$ & $82(55,4)$ & $38(40)$ & $217(47,8)$ \\
\hline $8-10$ & $4(3,9)$ & $16(14,8)$ & $20(13,5)$ & $33(34,7)$ & $73(16,1)$ \\
\hline Desconocido & $4(3,9)$ & $4(3,7)$ & $7(4,7)$ & $12(12,6)$ & $27(5,9)$ \\
\hline \multicolumn{6}{|c|}{ Factores pronósticos por PSA y Gleason } \\
\hline \multicolumn{6}{|c|}{ Buen pronóstico (PSA $<10$ y Gleason $<8$ ) } \\
\hline & $42(40,8)$ & $58(53,7)$ & $49(33,1)$ & $8(8,4)$ & $157(34,6)$ \\
\hline \multicolumn{6}{|c|}{ Mal pronóstico (PSA >=10 y Gleason $>7$ ) } \\
\hline & $1(1)$ & $6(5,6)$ & $11(7,4)$ & $27(28,4)$ & $45(9,9)$ \\
\hline \multicolumn{6}{|c|}{ Mal pronóstico (PSA >=20 y Gleason >7) } \\
\hline & $0(0)$ & $3(2,8)$ & $2(1,4)$ & $19(20)$ & $24(5,3)$ \\
\hline \multicolumn{6}{|c|}{ Factores pronósticos por PSA, Gleason y estadio } \\
\hline \multicolumn{6}{|c|}{ Buen pronóstico (PSA <10, Gleason $<7$ y Estadio = Localizado) } \\
\hline & $39(37,9)$ & $45(41,7)$ & $40(27)$ & $2(2,1)$ & $124(27,3)$ \\
\hline \multicolumn{6}{|c|}{ Mal pronóstico (PSA $>=10$, Gleason $>=7$ y Estadio $=$ No localizado) } \\
\hline & $35(34)$ & $1(0,9)$ & $85(57,4)$ & $70(73,7)$ & $246(54,2)$ \\
\hline \multicolumn{6}{|c|}{ Seguimiento vivos (años) } \\
\hline Media (IC) & $7(6,2-7,9)$ & $5,1(4,6-5,6)$ & $6,2(5,6-6,7)$ & $6,8(5,9-7,6)$ & $6,1(5,8-6,5)$ \\
\hline Mediana & 7 & 4,5 & 5,8 & 6,7 & 5,8 \\
\hline
\end{tabular}

hormonal (integrado sobre todo por enfermos con factores pronósticos desfavorables) en relación con el resto $(\mathrm{p}=0,00)$.

En los pacientes tratados con prostatectomía radical, antes de la cirugía eran tumores localizados 104 pacientes $(96,3 \%)$ y 4 localmente avanzados $(3,7 \%)$. Tras la cirugía fueron localizados 61 pacientes $(56,5 \%), 44(40,7 \%)$ localmente avanzados y $3(2,8 \%)$ diseminados
(3 pacientes con ganglios positivos en la pieza en formol). Estas diferencias en la proporción de tumores localizados y localmente avanzados antes y después de la cirugía es muy significativa $(p=0,00)$. De la misma forma esta proporción de resultados se ha mantenido a lo largo de todo el periodo del estudio, no habiendo diferencias significativas entre las distintas épocas $(\mathrm{p}=\mathrm{NS})$. 
En estos pacientes sometidos a cirugía hemos encontrado un subestadiaje clínico al diagnóstico del $41,7 \%$ (45 pacientes). Hemos encontrado márgenes positivos en 43 pacientes $(39,8 \%)$, en una proporción que se ha mantenido a lo largo de las distintas épocas, no habiéndose reducido en las cirugías realizadas en los últimos años ( $\mathrm{p}=\mathrm{NS}$ ).

\section{SUPERVIVENCIA LIBRE DE PROGRESIÓN}

En la Tabla 2 se expresan los datos de la SLP en los distintos grupos y en la Figura 1 se muestran en forma de curvas de Kaplan y Meier. En estas curvas al realizar el análisis log-rank no hemos encontrado diferencias significativas entre los tratados con cirugía y los tratados con radioterapia. Las diferencias con los otros grupos terapéuticos no son valorables por ser grupos no homogéneos.

La Tabla 3 refleja los resultados en cada grupo terapéutico del análisis de los factores pronósticos de cara a la SLP, tanto en análisis univariante como multivariante.
Al comparar entre los distintos grupos no hemos encontrado diferencias significativas en ninguno de los factores analizados salvo entre los pacientes tratados con cirugía y los tratados con radioterapia en donde la SLP ha sido superior en los tratados con radioterapia en aquellos pacientes en los que el PSA al diagnóstico ha sido igual o superior a $20 \mathrm{ng} / \mathrm{ml}(\mathrm{p}=0,01)$.

En el grupo de pacientes con PSA menor de 10, Gleason menor de 7 y estadio localizado (Fig. 2) y en los pacientes con PSA menor de $10 \mathrm{y}$ Gleason menor de 8, no hemos encontrado diferencias significativas entre los pacientes tratados con observación, cirugía o radioterapia.

\section{SUPERVIVENCIA GLOBAL}

Reflejamos en la Tabla 2 y en la Figura 3 los datos y las curvas actuariales de la SG. En el análisis de las curvas tampoco ha habido diferencias significativas en la supervivencia entre los pacientes tratados con cirugía y los tratados con radioterapia. En los pacientes tratados con bloqueo hormonal la SG ha sido menor $(\mathrm{p}=0,00)$.

Tabla 2. Resultados de la supervivencia libre de progresión, supervivencia global y supervivencia específica en cada uno de los grupos analizados

\begin{tabular}{|c|c|c|c|c|c|}
\hline & $\begin{array}{c}\text { Observación } \\
(\mathbf{n}=103)\end{array}$ & $\begin{array}{l}\text { Prostatectomia } \\
\qquad(\mathbf{n}=108)\end{array}$ & $\begin{array}{c}\text { Radioterapia } \\
\text { hormonal } \\
(\mathbf{n}=148)\end{array}$ & $\begin{array}{c}\text { Bloqueo } \\
(n=95)\end{array}$ & $\begin{array}{c}\text { Todos } \\
(n=454)\end{array}$ \\
\hline \multicolumn{6}{|c|}{ Supervivencia libre progresión (SLP) } \\
\hline Pacientes en progresión (\%) & $27(26)$ & $34(31)$ & $60(41)$ & $24(22)$ & $145(32)$ \\
\hline SLP a 5 años en \% & 77 & 61 & 63 & 73 & 68 \\
\hline SLP a 10 años & 67 & 50 & 25 & 67 & 50 \\
\hline SLP a 15 años & 45 & & & 67 & 38 \\
\hline Mediana & 12,7 & 7,8 & 6,8 & & 9,8 \\
\hline \multirow{4}{*}{$\begin{array}{ll}\text { SLP (Gleason 2-4) } & \text { a } 5 \text { años } \\
& \text { a } 10 \text { años } \\
& \text { a } 15 \text { años } \\
\text { SLP (Gleason 8-10) } & \text { a } 5 \text { años }\end{array}$} & 89 & 77 & 75 & 78 & 82 \\
\hline & 77 & & 22 & 78 & 57 \\
\hline & 67 & & & & 62 \\
\hline & & 36 & 38 & 63 & 53 \\
\hline \multicolumn{6}{|l|}{ Supervivencia global (SG) } \\
\hline Pacientes fallecidos (\%) & $32(31)$ & $14(13)$ & $33(22)$ & 47 (49) & $126(38)$ \\
\hline SG a 5 años en \% & 80 & 90 & 85 & 64 & 80 \\
\hline SG a 10 años & 61 & 76 & 67 & 32 & 59 \\
\hline SG a 15 años & 51 & & 43 & 32 & 44 \\
\hline Mediana & 19,1 & & 12,5 & 6,6 & 12,4 \\
\hline \multicolumn{6}{|l|}{ Supervivencia específica (SE) } \\
\hline Fallecidos por su tumor (\%) & $2(2)$ & $2(1,9)$ & $11(7,3)$ & $16(17)$ & $31(6,8)$ \\
\hline SE a 5 años en \% & 100 & 98 & 97 & 83 & 95 \\
\hline SE a 10 años & 94 & 98 & 88 & 77 & 89 \\
\hline SE a 15 años & 94 & & 67 & 77 & 78 \\
\hline
\end{tabular}




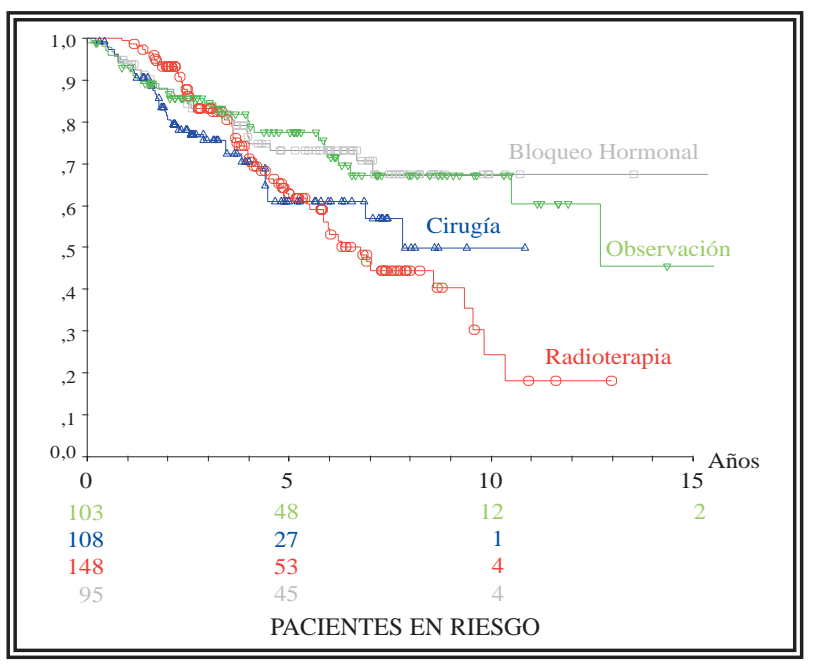

FIGURA 1. Curvas de supervivencia libre de progresión de cada grupo de tratamiento. Entre los pacientes tratados con cirugia y los tratados con radioterapia no hay diferencias significativas $(p=0,91)$.

En la Tabla 4 exponemos los resultados del análisis univariante y multivariante de los factores pronósticos en la $\mathrm{SG}$, realizado en cada grupo terapéutico.
Al comparar la SG en función de los distintos factores pronósticos entre los pacientes tratados con observación, cirugía y radioterapia no hemos encontrado diferencias significativas entre los tres grupos.

\section{SUPERVIVENCIA ESPECÍFICA}

La Tabla 2 y la Figura 4 expresan los datos y las curvas de supervivencia actuariales relativos a la $\mathrm{SE}$, los pacientes tratados con bloqueo hormonal han tenido una SE menor $(\mathrm{p}=0,03)$. Dado el escaso número de pacientes fallecidos por su tumor, tanto en el grupo de pacientes sometidos a observación, como en los tratados con prostatectomía radical, no se pueden hacer análisis de factores pronósticos en estos grupos ni comparativos con otros grupos.

En la Tabla 5 reflejamos los datos del análisis uni y multivariante de los factores pronósticos realizados en los pacientes tratados con radioterapia y los tratados con bloqueo hormonal.

Tabla 3. Supervivencia libre de progresión: análisis uni y multivariante de los factores pronósticos analizados

\begin{tabular}{|c|c|c|c|c|c|}
\hline & $\begin{array}{l}\text { Observación } \\
\qquad(n=103)\end{array}$ & $\begin{array}{l}\text { Prostatectomia } \\
\qquad(\mathrm{n}=108)\end{array}$ & $\begin{array}{l}\text { Radioterapia } \\
\qquad(\mathrm{n}=148)\end{array}$ & $\begin{array}{c}\text { Bloqueo } \\
\text { hormonal } \\
(n=95)\end{array}$ & $\begin{array}{l}\text { Todos } \\
(\mathbf{n}=454)\end{array}$ \\
\hline \multicolumn{6}{|l|}{ Factores pronósticos (univariante) } \\
\hline Edad & NS & NS & NS & Peor $<70(\mathrm{p}=0,03)$ & NS \\
\hline PSA al diagnóstico & NS & PSA $>=20(p=0,00)$ & PSA $>=20(p=0,04)$ & PSA $>=20(p=0,00)$ & PSA $>=20(p=0,00)$ \\
\hline Gleason & leason $<5(\mathrm{p}=0,00)$ & Gleason $>7(\mathrm{p}=0,00)$ & Gleason $>7(\mathrm{p}=0,04)$ & Gleason $>7(\mathrm{p}=0,02)$ & Gleason $>7(\mathrm{p}=0,02)$ \\
\hline Estadio $\quad M$ & Mejor T1 $(\mathrm{p}=0,00)$ & $\mathrm{NS}(\mathrm{p}=0,06)$ & Peor T3 $(\mathrm{p}=0,02)$ & Peor T3-T4 $(\mathrm{p}=0,00)$ & Peor T3-T4 $(\mathrm{p}=0,00)$ \\
\hline Localizado o localmente avanzado & o No valorable & No valorable & Mejor local. $(\mathrm{p}=0,00)$ & Mejor local. $(\mathrm{p}=0,00)$ & Mejor local. $(\mathrm{p}=0,00)$ \\
\hline Diagnóstico antes/después de 199 & $995 \quad$ NS & NS & NS & NS & NS \\
\hline $\begin{array}{l}\text { Factores buen pronóstico: } \\
\text { (PSA }<10 \text {, Gleason }<7 \text { y Est.=locali }\end{array}$ & lizado) $^{\text {NS }}$ & $\mathrm{p}=0,00$ & NS & No valorable & $\mathrm{p}=0,01$ \\
\hline $\begin{array}{l}\text { Factores mal pronóstico: } \\
(\text { PSA >=10 y Gleason }>7 \text { ) }\end{array}$ & NS & $\mathrm{p}=0,04$ & $\mathrm{p}=0,01$ & NS & $\mathrm{p}=0,01$ \\
\hline \multicolumn{6}{|c|}{ Análisis multivariante (Cox) y riesgo relativo (RR) con IC al $\mathbf{9 5 \%}$} \\
\hline $\begin{array}{l}\text { Primer factor } \\
\mathrm{RR}\end{array}$ & $\begin{array}{c}\text { Gleason }<\mathbf{5} \\
\text { RR: } 2,1 ; \text { IC: } 1,2-3,7\end{array}$ & $\begin{array}{c}\text { PSA >=20 } \\
\text { RR: } 3,3 ; \text { IC: } 2,1-4,8\end{array}$ & Ninguno & $\begin{array}{c}\text { Gleason }>\mathbf{7} \mathbf{y} \\
\text { PSA }>\mathbf{= 2 0} \\
\text { RR: } 7,3 ; \text { IC: } 1,3-27\end{array}$ & $\begin{array}{c}\text { Gleason }>\mathbf{7} \mathbf{~} \\
\text { PSA }>\mathbf{= 2 0} \\
\text { RR: } 2,3 ; \text { IC: } 1,4-3,6\end{array}$ \\
\hline Segundo factor & & 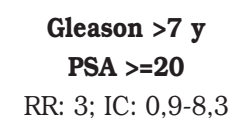 & & $\begin{array}{c}\text { PSA >=20 } \\
\text { RR: } 4 ; \text { IC: } 1,4-9,6\end{array}$ & $\begin{array}{c}\text { PSA >=20 } \\
\text { RR: } 1,9 ; \text { IC: } 1,4-2,6\end{array}$ \\
\hline Tercer factor & & & & & $\begin{array}{c}\text { Localmente } \\
\text { Avanzado } \\
\text { RR: } 1,7 ; \text { IC: } 1,4-2,2\end{array}$ \\
\hline
\end{tabular}




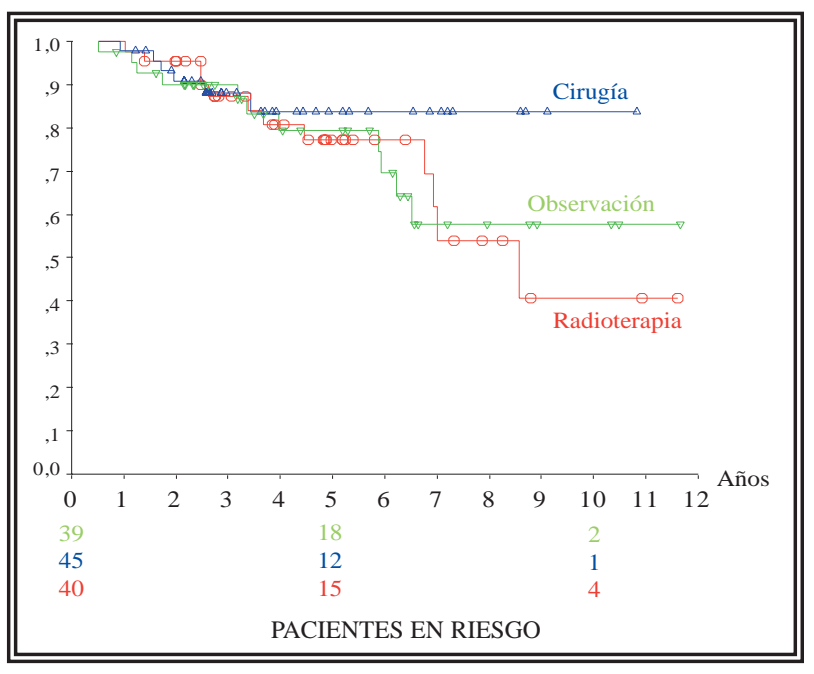

FIGURA 2. Curvas de supervivencia libre de progresión en los pacientes con factores de buen pronóstico (PSA menor de 10, Gleason menor de 7 y estadio localizado). No se incluyen los 2 pacientes tratados con bloqueo hormonal. Entre los 3 grupos analizados no ha habido diferencias significativas $(p=0,32)$.

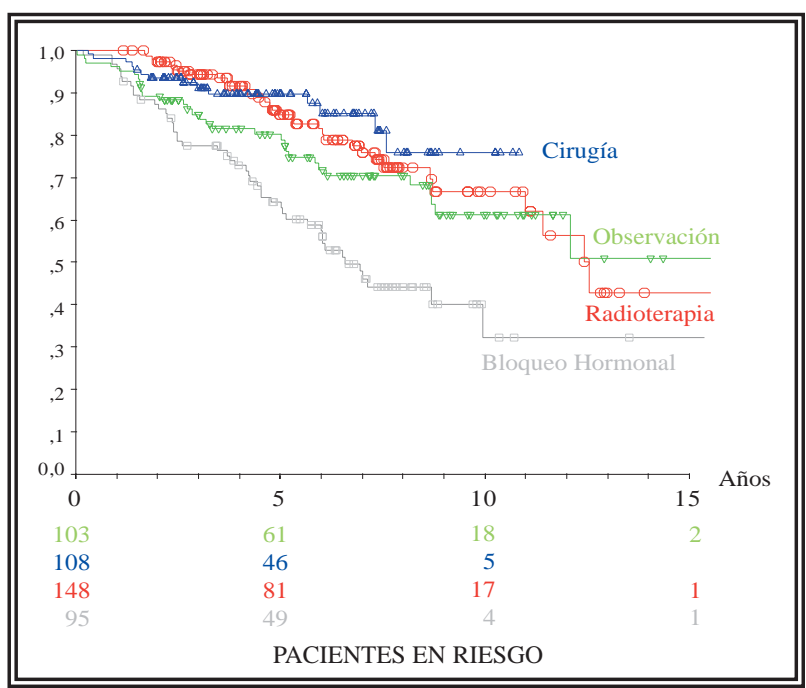

FIGURA 3. Curvas de supervivencia global. Entre los tratados con cirugía y los tratados con radioterapia no ha habido diferencias significativas $(p=0,54)$. La SG de los tratados con bloqueo hormonal ha sido peor $(p=0,00)$.

\section{REPERCUSIÓN DE LA PROGRESIÓN INICIAL EN LA SUPERVIVENCIA GLOBAL}

Las curvas de la Figura 5 muestran la SG de los pacientes que han entrado en progresión frente a los pacientes sin progresión de toda la muestra, no ha habido diferencias significativas. En los pacientes tratados con observación y en los tratados con radioterapia tampoco ha habido diferencias estadísticamente significativas.
Los enfermos tratados con prostatectomía radical que han entrado en progresión han tenido una SG superior frente a los que no $(p=0,01)$ (Fig. 6). Los pacientes tratados con bloqueo hormonal que han progresado han tenido una SG inferior a los que no $(\mathrm{p}=0,00)$.

\section{DISCUSIÓN}

El antígeno prostático específico ha hecho que se traslade el diagnóstico del cáncer de próstata a estadios precoces, siendo sin duda el mejor marcador para el diagnóstico de esta enfermedad $^{5}$. En la actualidad en nuestro medio una importante parte de las determinaciones de PSA realizadas a pacientes nuevos se realizan en diversos chequeos rutinarios. Su utilización generalizada ha producido un incremento real en la incidencia de esta enfermedad. A pesar de la gran incidencia y prevalencia de este tumor desconocemos cual es su mejor manejo.

Al analizar los resultados obtenidos en la bibliografía sobre cáncer de próstata localizado utilizando distintas alternativas terapéuticas, observamos que existen importantes diferencias entre las series.

\section{TRATAMIENTO CONSERVADOR DEL CÁNCER DE PRÓSTATA}

La alternativa observacional diferentes autores la aconsejan en tumores de bajo grado ${ }^{6,7}, \sin$ embargo existen numerosas series publicadas con este manejo conservador y no sólo en tumores de buen pronóstico ${ }^{8-10}$. En 1994 Chodak publica una recopilación de 10 series con 828 pacientes sometidos a observación ${ }^{11}$. Los resultados obtenidos por este autor en tumores de bajo grado (Gleason 2-4), evaluando la supervivencia libre de progresión, son muy parecidos a los nuestros: a 5 años 90\% (nosotros 89\%), a 10 años $81 \%$ (77\%), a 15 años 60\% (67\%).

La supervivencia específica de nuestra serie es algo superior a la de otras series publicadas. Nosotros hemos obtenido un 100\% de SE a 5 años y del $94 \%$ a 10 y 15 años. Chodak obtiene en tumores de bajo grado a 5 años un $100 \%$ y a 15 años un $60 \%$. Johansson a 15 años obtiene un $81 \%^{10}$. En cualquier caso estos datos objetivan una evolución bastante homogénea de los cánceres de próstata localizados sometidos a observación. 
Tabla 4. Supervivencia global: análisis uni y multivariante de los factores pronósticos analizados

\begin{tabular}{|c|c|c|c|c|c|}
\hline & $\begin{array}{c}\text { Observación } \\
\qquad(n=103)\end{array}$ & $\begin{array}{l}\text { Prostatectomía } \\
\qquad(\mathrm{n}=108)\end{array}$ & $\begin{array}{c}\text { Radioterapia } \\
\qquad(\mathrm{n}=148)\end{array}$ & $\begin{array}{c}\text { Bloqueo } \\
\text { hormonal } \\
(n=95)\end{array}$ & $\begin{array}{l}\text { Todos } \\
(n=454)\end{array}$ \\
\hline \multicolumn{6}{|l|}{ Factores pronósticos (univariante) } \\
\hline Edad & Peor $>75(\mathrm{p}=0,00)$ & Peor $>70(\mathrm{p}=0,01)$ & Peor $>75(\mathrm{p}=0,04)$ & Peor $>75(\mathrm{p}=0,04)$ & Peor $>75(\mathrm{p}=0,00)$ \\
\hline PSA al diagnóstico & NS & NS & NS & PSA $>=20(p=0,01)$ & PSA $>=20(p=0,00)$ \\
\hline Gleason & NS & Mejor $\mathrm{G}<8(\mathrm{p}=0,02)$ & NS & NS & Gleason $>7(\mathrm{p}=0,02)$ \\
\hline Estadio & Peor T3 $(\mathrm{p}=0,02)$ & NS & NS & NS & Peor T3-T4 $(\mathrm{p}=0,00)$ \\
\hline Localizado o localmente avanzado $\mathrm{P}$ & Peor loc. av. $(\mathrm{p}=0,00)$ & No valorable & NS & Mejor local. $(\mathrm{p}=0,02)$ & Mejor local. $(\mathrm{p}=0,00)$ \\
\hline Diagnóstico antes/después de 1995 & $5 \quad \mathrm{NS}$ & NS & NS & NS & NS \\
\hline $\begin{array}{l}\text { Factores buen pronóstico: } \\
\text { (PSA }<10 \text {, Gleas. }<7 \text { y Est. = Localiz.) }\end{array}$ & NS & NS & NS & NS & NS \\
\hline $\begin{array}{l}\text { Factores mal pronóstico: } \\
(\text { PSA }>=10 \text { y Gleason }>7)\end{array}$ & NS & NS & NS & NS & $\mathrm{p}=0,02$ \\
\hline $\begin{array}{l}\text { Factores mal pronóstico: } \\
(\mathrm{PSA}>=20 \text { y Gleason }>7)\end{array}$ & NS & NS & No valorable & NS & $\mathrm{p}=0,00$ \\
\hline \multicolumn{6}{|c|}{ Análisis multivariante (Cox) y riesgo relativo (RR) con IC al 95\% } \\
\hline Primer factor & $\begin{array}{l}\text { Edad (variable } \\
\text { continua) }\end{array}$ & $\begin{array}{c}\text { Edad (variable } \\
\text { continua) }\end{array}$ & $\begin{array}{l}\text { Edad (variable } \\
\text { continua) }\end{array}$ & $\begin{array}{c}\text { Edad y PSA } \\
\text { (variables continuas) }\end{array}$ & $\begin{array}{c}\text { Edad y PSA } \\
\text { (variables continuas) }\end{array}$ \\
\hline Segundo factor & & & & $\begin{array}{c}\text { PSA >=20 } \\
\text { RR: } 1,5 ; \text { IC: } 0,9-2,4\end{array}$ & $\begin{array}{c}\text { Gleason }>7 \mathrm{y} \\
\text { PSA }>=20\end{array}$ \\
\hline & & & & & RR: 2,98 ; IC: $1,8-4,9$ \\
\hline Tercer factor & & & & $\begin{array}{c}\text { Localmente } \\
\text { avanzado } \\
\text { RR: } 1,5 ; \text { IC: } 1-2,2\end{array}$ & $\begin{array}{c}\text { Localmente } \\
\text { avanzado } \\
\text { RR: } 2,1 ; \text { IC: } 1,5-2,8\end{array}$ \\
\hline
\end{tabular}

\section{PROSTATECTOMÍA RADICAL}

La prostatectomía radical es probablemente la alternativa terapéutica más utilizada por los urólogos en el cáncer de próstata localizado ${ }^{12}$. Los factores pronósticos condicionantes de la progresión son aquellos que reflejan la carga tumoral del paciente. Los más importantes son: PSA al diagnóstico, estadio, Gleason y existencia de márgenes positivos ${ }^{13-23}$. Nuestros resultados, en los factores que hemos analizado, coinciden con la bibliografia.

$\mathrm{Al}$ evaluar la supervivencia libre de progresión existen diferencias entre las distintas series publicadas. Nosotros a 5 años hemos obtenido un $61 \%$ y a 10 años un $50 \%$, estos resultados coinciden con otras series ${ }^{15,24}$. Sin embargo, existen series con resultados muy superiores ${ }^{21,25-27}$. Pound y cols. ${ }^{25}$ a 5 años tienen un $84 \%$ de SLP, Hull y cols. ${ }^{21}$ a 10 años obtienen un $75 \%$.

En pacientes con Gleason elevado (8-10) hemos obtenido a 5 años una SLP de $36 \%$, Hull y cols. ${ }^{21}$ en una serie de 1.000 prostatectomías radicales obtienen un $49 \%$ a 5 años en este grupo.

En nuestra serie no hemos encontrado diferencias en la SLP al comparar los resultados obtenidos en las primeras 50 prostatectomías radicales con las 58 siguientes del estudio.

La supervivencia específica que hemos obtenido a 5 y 10 años ha sido del 98\%, semejante a otras series americanas ${ }^{24,27}$ y superior al $76 \%$ obtenido por Rioja y cols. ${ }^{28}$

\section{TRATAMIENTO RADIOTERÁPICO}

Al analizar los resultados obtenidos con el tratamiento radioterápico en el cáncer de próstata también encontramos diferencias entre los distintos autores. Nosotros hemos obtenido un 63\% de SLP a 5 años y un $25 \%$ a 10 años, estas cifras son semejantes a las obtenidas por Zapatero y cols. en nuestro país (SLP a 3 años $74 \%)^{29}$ y algo inferiores a las obtenidas por Zietman y cols. a 10 años $(40 \%)^{30}$. 
Los factores pronósticos analizados confirman que la carga tumoral (PSA, Gleason, estadio, etc.) son los factores más importantes de cara a la SLP coincidiendo con otras series de la bibliografia $^{31,32}$. En los pacientes con Gleason elevado la SLP a 5 años ha sido de $38 \%$, semejante a la obtenida en los pacientes operados (36\%).

Hemos encontrado una supervivencia global (SG) a 5 años del 85\%, a 10 años del $67 \%$ y a 15 años del $43 \%$. La supervivencia específica (SE) a 5 años ha sido del 97\%, a 10 años del $88 \%$ y a 15 del 67\%. Nuestros resultados a 15 años tanto en la SG como en la SE son muy similares a los obtenidos por Gray y cols. que encuentran un $46 \%$ de $\mathrm{SG}$ y un $64,5 \%$ de $\mathrm{SE}^{33}$.

En nuestra serie al analizar los factores pronósticos en la supervivencia específica ningún factor ha tenido significación estadística, probablemente debido al bajo número de pacientes fallecidos por el cáncer de próstata: de los 148 pacientes tratados con radioterapia han fallecido 33 y de éstos sólo 11 por su tumor. Otras series encuentran un incremento en la mortalidad específica cuando se incrementan los factores de riesgo tumorales ${ }^{34,35}$.

\section{TRATAMIENTO HORMONAL}

En la actualidad no utilizamos el tratamiento hormonal como primera medida terapéutica en el cáncer de próstata localizado, lo utilizamos cuando existe progresión con sintomatología clínica. Sin embargo estudios recientes encuentran un incremento de la SLP utilizando un antiandrógeno sólo o en adyuvancia con otros tratamientos ${ }^{36}$. Nosotros hemos encontrado una SE menor que en los otros grupos.

\section{ESTUDIOS COMPARATIVOS}

No existen datos concluyentes que avalen una determinada alternativa de tratamiento como la mejor opción terapéutica. Existen muchas series retrospectivas que analizan los resultados comparativos de distintos tratamientos. Stokes compara tres series de pacientes tratados con prostatectomía radical, radioterapia o braquiterapia; en pacientes de bajo riesgo no encuentra diferencias significativas en la SLP a 5 años entre los 3 grupos (en torno al 70\%). En los pacientes de alto riesgo encuentra mayor SLP en los tratados con prostatectomía que en los otros dos grupos ${ }^{37}$. Kupelian y cols. ${ }^{38}$ encuentran los mismos resultados al comparar pacientes tratados con radioterapia o con prostatectomía radical.

Recientemente D'Amico y cols. ${ }^{39}$ en una amplia serie de 2.635 pacientes tratados con prostatectomía radical (2.254 pacientes) o con radioterapia (381 pacientes), encuentran resultados contrarios a los anteriores. En pacientes con tumores de bajo riesgo tienen mejor SLP los tratados con cirugía que los tratados con radioterapia. Por el contrario en los pacientes de alto riesgo tienen mejor SLP los tratados con radioterapia hasta el octavo año, a partir de este año las dos alternativas presentan igual SLP.

Thompson y cols. ${ }^{40}$ al analizar 1.286 pacientes con cáncer de próstata metastásico tratados con bloqueo hormonal, encuentran una supervivencia global superior en los pacientes en los que el primer tratamiento fue prostatectomía que en los que fue radioterapia.

Existen dificultades para realizar estudios randomizados que evalúen estas alternativas terapéuticas sobre todo para aceptar el brazo de observación. Ésta es la razón por la que en el Reino Unido el North-West Uro-Oncology Group acepte agrupar los pacientes por distintos tratamientos siempre que lo haya escogido el propio paciente ${ }^{41}$.

Dentro de estos escasos ensayos clínicos son importantes los resultados obtenidos por Holmberg y cols. publicados en el año $2002^{42}$. Es un estudio multicéntrico con un total de 698 pacientes con tumor localizado. Son randomizados a observación (349 pacientes) o prostatectomía (349 pacientes). Encuentran una diferencia significativa en la supervivencia específica favorable a los pacientes tratados con cirugía frente a los sometidos a observación. Sin embargo, no encuentran diferencias entre las dos alternativas al evaluar la supervivencia global.

En nuestra experiencia, dentro de las limitaciones de un estudio retrospectivo y con grupos no homogéneos, no hemos encontrado diferencias en la supervivencia libre de progresión entre los pacientes tratados con radioterapia y los tratados con prostatectomía radical, tanto al analizarlos en forma global (Fig. 1) como en función de los distintos factores pronósticos, salvo en los casos en que el PSA ha sido superior a 20 en 
donde la SLP ha sido significativamente mejor en los tratados con radioterapia que en los enfermos operados $(\mathrm{p}=0,00)$.

$\mathrm{Al}$ analizar la supervivencia global de nuestra serie no ha habido diferencias significativas entre los distintos grupos, salvo en los tratados con bloqueo hormonal en los que la SG ha sido menor probablemente por tener la edad media más elevada (Fig. 3).

De los 126 pacientes que han fallecido en nuestra serie sólo 31 (25\%) lo han sido por su cáncer de próstata. Esto hace que la supervivencia específica (SE) sea muy elevada en todos los grupos terapéuticos. Al comparar estos grupos de forma global no hemos encontrado diferencias significativas entre los tratados con observación, radioterapia o cirugía. Tampoco hemos encontrado diferencias al estratificar por factores pronósticos. Los tratados con bloqueo hormonal han tenido una SE peor (Fig. 4).

\section{IMPACTO DE LA PROGRESIÓN INICIAL EN LA SUPERVIVENCIA}

En el año 2002 Kupelian y cols. ${ }^{43}$ analizan en una serie de 936 pacientes tratados con radioterapia externa la repercusión en la supervivencia global de la progresión bioquímica en los primeros 10 años tras el tratamiento. Encuentran progresión en 316 pacientes (34\%), no encontrando diferencias en la supervivencia global entre los

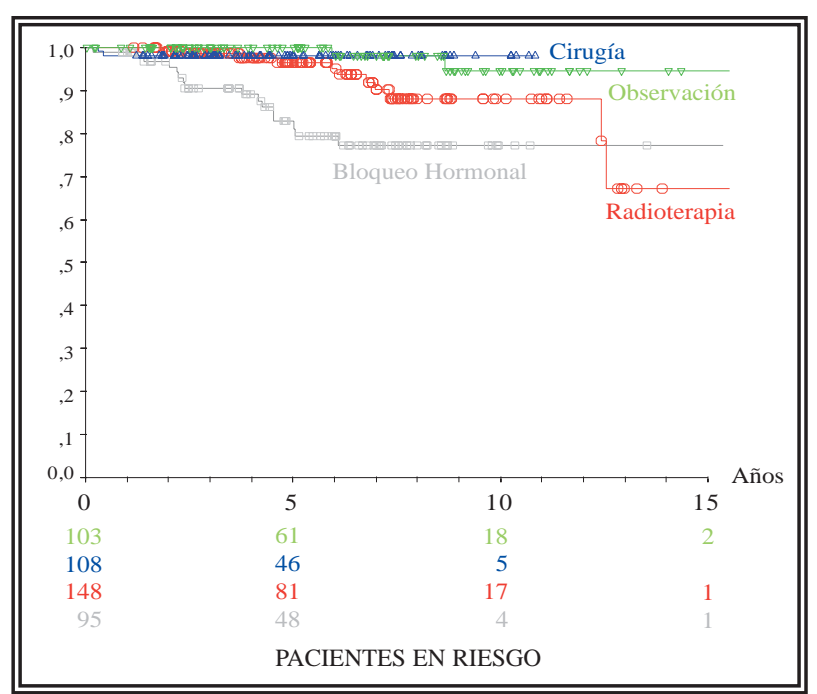

FIGURA 4. Curvas de supervivencia especifica. Por el escaso número de pacientes fallecidos por su tumor en los tratados con observación y los tratados con cirugía no se pueden hacer estudios comparativos.

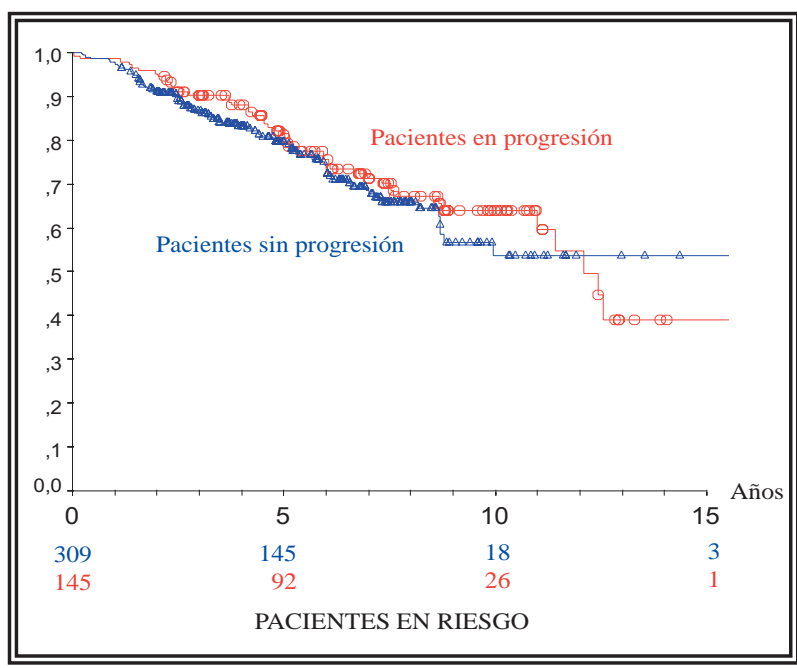

FIGURA 5. Curvas de supervivencia global de todos los grupos analizados en conjunto en función de si los pacientes han entrado en progresión. No ha habido diferencias significativas $(p=0,42)$.

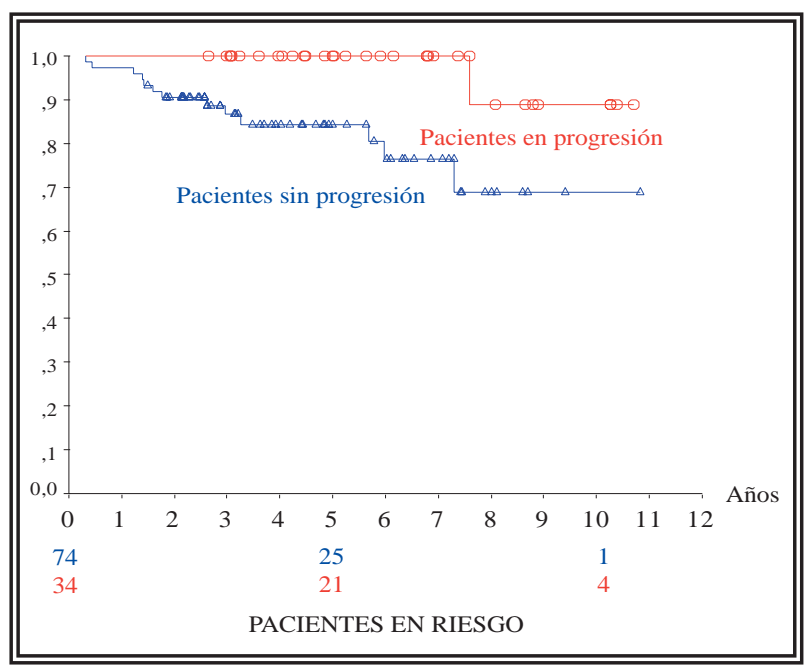

FIGURA 6. Curvas de supervivencia global de los pacientes sometidos a prostatectomia radical en función de si han entrado en progresión. Los pacientes que han progresado han tenido una $\mathbf{S G}$ superior $(p=0,01)$.

que han entrado en progresión y los que no han progresado en los 10 primeros años. Concluyen considerando que esta diferencia pueda aparecer a más largo plazo, a 15 ó 20 años.

Nuestra experiencia confirma estos resultados no sólo en los tratados con radioterapia sino también en los sometidos a observación y en los tratados con prostatectomía radical. En los tratados con bloqueo hormonal la supervivencia de los pacientes que progresaron ha sido menor con significación estadística. 
Tabla 5. Supervivencia específica: análisis uni y multivariante de los factores pronósticos analizados en los pacientes tratados con radioterapia y los tratados con bloqueo hormonal

\begin{tabular}{|c|c|c|}
\hline & $\begin{array}{l}\text { Radioterapia } \\
(\mathrm{n}=148)\end{array}$ & $\begin{array}{l}\text { Bloqueo hormonal } \\
\qquad(\mathrm{n}=95)\end{array}$ \\
\hline \multicolumn{3}{|l|}{ Factores pronósticos (univariante) } \\
\hline Edad & NS & NS \\
\hline PSA al diagnóstico & NS & PSA $>=20(p=0,00)$ \\
\hline Gleason & NS & NS \\
\hline Estadio & NS & Peor T3-T4 $(\mathrm{p}=0,02)$ \\
\hline Localizado o localmente avanzado & NS & Mejor local. $(\mathrm{p}=0,00)$ \\
\hline Diagnóstico antes/después de 1995 & NS & Peor antes $1995(\mathrm{p}=0,02)$ \\
\hline $\begin{array}{l}\text { Factores buen pronóstico: } \\
\text { (PSA }<10 \text {, Gleas. }<7 \text { y Est. = Localiz.) }\end{array}$ & NS & NS \\
\hline $\begin{array}{l}\text { Factores mal pronóstico: } \\
(\text { PSA >=10 y Gleason }>7)\end{array}$ & NS & NS \\
\hline $\begin{array}{l}\text { Factores mal pronóstico: } \\
\text { (PSA >=20 y Gleason > } 7 \text { ) }\end{array}$ & NS & $\mathrm{p}=0,00$ \\
\hline \multicolumn{3}{|l|}{$\begin{array}{l}\text { Análisis multivariante (Cox) y } \\
\text { riesgo relativo (RR) con IC al } 95 \%\end{array}$} \\
\hline Primer factor & & PSA (variable continua) \\
\hline Segundo factor & & $\begin{array}{l}\text { Localmente avanzado } \\
\text { RR: } 6,1 ; \text { IC: } 1,9-15,3\end{array}$ \\
\hline
\end{tabular}

En los pacientes tratados con cirugia la SG ha sido superior en los que han progresado frente a los que no. Es posible que ésto se deba al escaso número de pacientes fallecidos (14 de 108) y que al haberse producido de una forma precoz (antes de los 10 años tras cirugía) no haya dado tiempo a la entrada en progresión.

\section{OTROS ASPECTOS EN EL CÁNCER DE PRÓSTATA LOCALIZADO}

La utilización de otras alternativas terapéuticas actualmente en uso es motivo de controversia. Aus y cols. ${ }^{44}$ en un estudio prospectivo evaluando los resultados de la crioterapia aconsejan abandonar esta técnica por las muchas complicaciones y escasos logros oncológicos que encuentran. Como alternativa a las 3 opciones clásicas: observación, prostatectomía radical o tratamiento radioterápico, Leibowitz y Tucker ${ }^{45}$ encuentran buenos resultados al tratar tumores localizados con un triple bloqueo androgénico: agonista LH-RH más un antiandrógeno más finasteride.

Como vemos las alternativas pueden ser múltiples, lo que demuestra lo lejos que estamos de tener una respuesta válida y eficaz. Nuestros pro- pios resultados no confirman una alternativa frente a otras. Todos los tratamientos intervencionistas tienen una elevada morbilidad que no hemos analizado en el estudio.

En el año 2002 Harris y Lohr del Research Triangle Institute-University of North Caroline Evidence-based Practice Center, examinan para la USPSTF (U.S. Preventive Services Task Force) la evidencia de los beneficios y daños del screening y tratamiento precoz del cáncer de prósta$\mathrm{ta}^{4}$. Estos autores tras analizar la bibliografía con los criterios de la medicina basada en la evidencia, concluyen que están establecidos los daños producidos por el tratamiento precoz del cáncer de próstata, pero no están claramente demostrados los potenciales beneficios. En base a estos hechos la USPSTF desaconseja la detección precoz del cáncer de próstata ${ }^{46}$.

Por otra parte el manejo de estos pacientes que han entrado en progresión no está bien definido. En el año 2002 Nair y cols. ${ }^{47}$ realizan una revisión de ensayos clínicos para la Cochrane Library, para valorar las ventajas e inconvenientes de la supresión androgénica precoz versus la tardía en el cáncer de próstata avanzado. Encuentran que 
el tratamiento precoz reduce la progresión de la enfermedad y las complicaciones asociadas a la progresión, pero se incrementan los efectos secundarios y los costes. Este tratamiento precoz produce un pequeño pero significativo incremento de la supervivencia global a 10 años, pero sin aumentar de forma estadísticamente significativa la supervivencia específica. En cualquier caso aconsejan estudios adicionales para evaluar mejor estos resultados.

\section{CONCLUSIONES}

En base a nuestros resultados y a la revisión bibliográfica realizada en el momento actual, no existe una alternativa terapéutica que ofrezca una superioridad frente a otros tratamientos en el cáncer de próstata localizado. Es preciso que se completen ensayos clínicos como el PIVOT (Prostate Intervention Versus Observation Trial) ${ }^{48}$ para poder tener datos de mayor fiabilidad, aunque esto puede tardar varios años. Como consecuencia hemos modificado nuestra actitud global en el cáncer de próstata tanto de cara al diagnóstico como en el tratamiento.

En adultos jóvenes antes de realizarles de forma sistemática una determinación de PSA, les informamos de las ventajas e inconvenientes que esta determinación puede producir. Sin embargo, en la actualidad esta medida tiene una efectividad relativa dada la presión social para realizar estas determinaciones.

A los pacientes diagnosticados de cáncer de próstata se les informa de las ventajas e inconvenientes de las distintas alternativas incluyendo la observación, para que posteriormente decidan el tratamiento. Sin embargo, la posibilidad de observación sobre todo en adultos jóvenes es una alternativa prácticamente no utilizada por dos razones: la dificultad para entender la realización de un diagnóstico oncológico si no es para hacer un tratamiento activo, así como por la propia carga emocional que el diagnóstico lleva consigo.

\section{REFERENCIAS}

1. HERRANZ AMO F, ARIAS FUNEZ F, ARRIZABALAGA MORENO $\mathrm{M}$ et al.: El cáncer de próstata en la Comunidad de Madrid en el año 2000. Monografía. 2002.

2. ARRIZABALAGA MORENO M, GARCÍA GONZÁLEZ JI, DÍEZ RODRÍGUEZ JM et al.: Indicadores epidemiológicos del adenocarcinoma de próstata. Resultados sobre 436 pacientes. Actas Urol Esp 1997; 21: 852-861.
3. ETZIONI R, PENSON DF, LEGLER JM et al.: Overdiagnosis due to prostate-specific antigen screening: lessons from U.S. prostate cancer incidence trends. J Natl Cancer Inst 2002; 94: 981-990.

4. HARRIS R, LOHR KN.: Screening for prostate cancer: an update of the evidence for the U.S. Preventive Services Task Force. Ann Intern Med 2002; 137: 917-929.

5. HAKAMA M, STENMAN UH, AROMAA A, LEINONEN J, HAKULINEN T, KNEKT P.: Validity of the prostate specific antigen test for prostate cancer screening: followup study with a bank of 21.000 sera in Finland. J Urol 2001; 166: 2189-2191.

6. SCHMID HP, ADOLFSSON J, AUS G: Active monitoring (deferred treatment or watchful waiting) in the treatment of prostate cancer. A review. Eur Urol 2001; 40: 488-494.

7. CARTER HB, WALSH PC, LANDIS P, EPSTEIN JI.: Expectant management of nonpalpable prostate cancer with curative intent: preliminary results. J Urol 2002; 167: 1231-1234.

8. SANDBLOM G, DUFMATS M, VARENHORST E.: Long-term survival in a Swedish population-based cohort of men with prostate cancer. Urology 2000; 56: 442-447.

9. BRASSO K, FRIIS S, JUEL K, JORGENSEN T, IVERSEN P.: The need for hospital care of patients with clinically localized prostate cancer managed by noncurative intent: a population based registry study. J Urol 2000; 163: 1150-1154.

10. JOHANSSON JE.: Natural disease course in untreated early prostatic carcinoma-the Orebro Study. Schweiz Rundsch Med Prax 2001; 90: 1507-1514.

11. CHODAK GW, THISTED RA, GERBER GS et al.: Results of conservative management of clinically localized prostate cancer. N Engl J Med 1994; 330: 242-248.

12. HANNA CL, MASON MD, DONOVAN JL, BARBER JP.: Clinical oncologists favour radical radiotherapy for localized prostate cancer: a questionnaire survey. BJU Int 2002; 90: 558-560.

13. CATALONA WJ, SMITH DS.: Cancer recurrence and survival rates after anatomic radical retropubic prostatectomy for prostate cancer: intermediate-term results. J Urol 1998; 160: 2428-2434.

14. ZUDAIRE BERGERA JJ, MARTÍN-MARQUINA ASPIUNZA A, SÁNCHEZ ZALABARDO D et al.: Prostatectomía radical en adenocarcinoma de próstata clínicamente localizado. Estudio de los pacientes con márgenes positivos y su influencia en la supervivencia libre de progresión bioquímica. Actas Urol Esp 1999; 23: 835-842.

15. ZUDAIRE BERGERA JJ, MARTÍN-MARQUINA ASPIUNZA A, SÁNCHEZ ZALABARDO D et al.: Prostatectomía radical en adenocarcinoma de próstata clínicamente localizado. Factores influyentes en la supervivencia libre de progresión bioquímica. Actas Urol Esp 1999; 23: 333-341.

16. GIBANEL GARANTO R, BEARDO VILLAR P, ALCARAZ ASENSIO A et al.: Significado de los márgenes positivos en la prostatectomía radical por cáncer localizado. Actas Urol Esp 2000; 25 (Suplemento LXV Congreso Nacional de Urologia): 34 (C 41).

17. ZUDAIRE BERGERA JJ, LÓPEZ FERRÁNDIZ J, SÁNCHEZ ZALABARDO D et al.: Prostatectomía radical en adenocarcinoma de próstata estadio pT3c. Actas Urol Esp 2000; 24: 468-474.

18. GIL MARTÍNEZ P, GIL SANZ MJ, ALLEPUZ LOSA C, BORQUE FERNANDO A, VALDIVIA NAVARRO P, RIOJA SANZ LA.: Cáncer de próstata estadio pT3 tras prostatectomía radical. Resultados en progresión y supervivencia. Actas Urol Esp 2000; 245: 400-405.

19. D'AMICO AV, WHITTINGTON R, MALKOWICZ SB et al.: Redicting prostate specific antigen outcome preoperatively in the prostate specific antigen era. J Urol 2001; 166: 21852188 . 
20. LAU WK, BERGSTRALH EJ, BLUTE ML, SLEZAK JM, ZINCKE H.: Radical prostatectomy for pathological Gleason 8 or greater prostate cancer: influence of concomitant pathological variables. J Urol 2002; 167: 117-122.

21. HULL GW, RABBANI F, ABBAS F, WHEELER TM, KATTAN MW, SCARDINO PT.: Cancer control with radical prostatectomy alone in 1.000 consecutive patients. J Urol 2002; 167: 528-534.

22. PALISAAN RJ, GRAEFEN M, KARAKIEWICZ PI et al. Assessment of clinical and pathologic characteristics predisposing to disease recurrence following radical prostatectomy in men with pathologically organ-confined prostate cancer. Eur Urol 2002; 41: 155-161.

23. HAN M, PARTIN AW, ZAHURAK M, PIANTADOSI S, EPSTEIN JI, WALSH PC.: Biochemical (prostate specific antigen) recurrence probability following radical prostatectomy for clinically localized prostate cancer. J Urol 2003; 169: 517-523.

24. ZINCKE H, OESTERLING JE, BLUTE ML, BERGSTRALH EJ, MYERS RP, BARRETT DM.: Long-term (15 years) results after radical prostatectomy for clinically localized (stage T2c or lower) prostate cancer. J Urol 1994; 152: 1850-1857.

25. POUND CR, PARTIN AW, EPSTEIN JI, WALSH PC.: Prostatespecific antigen after anatomic radical retropubic prostatectomy. Patterns of recurrence and cancer control. Urol Clin North Am 1997; 24: 395-406.

26. AMLING CL, BLUTE ML, BERGSTRALH EJ, SEAY TM, SLEZAK J, ZINCKE H.: Long-term hazard of progression after radical prostatectomy for clinically localized prostate cancer: continued risk of biochemical failure after 5 years. $J$ Urol 2000; 164: 101-105.

27. HAN M, PARTIN AW, POUND CR, EPSTEIN JI, WALSH PC.: Long-term biochemical disease-free and cancer-specific survival following anatomic radical retropubic prostatectomy. The 15-year Johns Hopkins experience. Urol Clin North Am 2001; 28: 555-565.

28. RIOJA SANZ LA, LIÉDANA TORRES JM, RONCALES BADAL A et al.: Análisis de una serie de prostatectomías radicales. Actas Urol Esp 1997; 21: 809-816.

29. ZAPATERO LABORDA A, LÓPEZ RODRÍGUEZ M, MÍNGUEZ MARTÍNEZ R, RODRÍGUEZ F, PÉREZ TORRUBIA A: Control bioquímico tras radioterapia externa en el cáncer de próstata localizado: resultados de una cohorte moderna. Actas Urol Esp 2000; 24: 10-18.

30. ZIETMAN AL, COEN JJ, DALlOW KC, SHIPLEY WU.: The treatment of prostate cancer by conventional radiation therapy: an analysis of long-term outcome. Int $J$ Radiat Oncol Biol Phys 1995 may 15; 32 (2): 287-292.

31. VALICENTI R, LU J, PILEPICH M, ASBELL S, GRIGNON D.: Survival advantage from higher-dose radiation therapy for clinically localized prostate cancer treated on the Radiation Therapy Oncology Group trials. J Clin Oncol 2000; 18: 27402746.

32. DO TM, PARKER RG, SMITH RB, KAGAN AR.: High-grade carcinoma of the prostate: a comparison of current local therapies. Urology 2001; 57: 1121-1126.

33. GRAY CL, POWELL CR, RIFFENBURGH RH, JOHNSTONE PA.: 20-year outcome of patients with T1-3NO surgically staged prostate cancer treated with external beam radiation therapy. J Urol 2001; 166: 116-118.

34. MAARTENSE S, HERMANS J, LEER JW.: Radiation therapy in localized prostate cancer: long-term results and late toxicity. Clin Oncol (R Coll Radiol) 2000; 12: 222-228.

35. ROACH M, LU J, PILEPICH MV et al.: Four prognostic groups predict long-term survival from prostate cancer following radiotherapy alone on Radiation Therapy Oncology Group clinical trials. Int J Radiat Oncol Biol Phys 2000; 47: 609-615.
36. SEE WA, WIRTH MP, MCLEOD DG et al.: Bicalutamide as immediate therapy either alone or as adjuvant to standard care of patients with localized or locally advanced prostate cancer: first analysis of the early prostate cancer program. $J$ Urol 2002; 168: 429-435.

37. STOKES SH.: Comparison of biochemical disease-free survival of patients with localized carcinoma of the prostate undergoing radical prostatectomy, transperineal ultrasoundguided radioactive seed implantation, or definitive external beam irradiation. Int J Radiat Oncol Biol Phys 2000; 47: 129136.

38. KUPELIAN P, KATCHER J, LEVIN H et al.: External beam radiotherapy versus radical prostatectomy for clinical stage T1-2 prostate cancer: therapeutic implications of stratification by pretreatment PSA levels and biopsy Gleason scores. Cancer J Sci Am 1997; 3: 78-87.

39. D'AMICO AV, WHITTINGTON R, MALKOWICZ SB et al.: Biochemical outcome after radical prostatectomy or external beam radiation therapy for patients with clinically localized prostate carcinoma in the prostate specific antigen era. Cancer 2002; 95: 281-286.

40. THOMPSON IM, TANGEN C, BASLER J, CRAWFORD ED.: Impact of previous local treatment for prostate cancer on subsequent metastatic disease. J Urol 2002; 168: 1008-1012.

41. THE NORTH-WEST URO-ONCOLOGY GROUP.: A preliminary report on a patient-preference study to compare treatment options in early prostate cancer. BJU Int 2002; 90: 253256.

42. HOLMBERG L, BILL-AXELSON A, HELGESEN F et al.: A randomized trial comparing radical prostatectomy with watchful waiting in early prostate cancer. N Engl J Med 2002; 347: 781-789.

43. KUPELIAN PA, BUCHSBAUM JC, PATEL C et al.: Impact of biochemical failure on overall survival after radiation therapy for localized prostate cancer in the PSA era. Int J Radiat Oncol Biol Phys 2002; 52: 704-711.

44. AUS G, PILEBLAD E, HUGOSSON J.: Cryosurgical ablation of the prostate: 5-year follow-up of a prospective study. Eur Urol 2002; 42: 133-138.

45. LEIBOWITZ RL, TUCKER SJ.: Treatment of localized prostate cancer with intermittent triple androgen blockade: preliminary results in 110 consecutive patients. Oncologist 2001; 6: 177-182.

46. U.S. PREVENTIVE SERVICES TASK FORCE.: Screening for prostate cancer: recommendation and rationale. Ann Intern Med 2002; 137: 915-916.

47. NAIR B, WILT T, MACDONALD R, RUTKS I.: Early versus deferred androgen suppression in the treatment of advanced prostatic cancer. Cochrane Database Syst Rev 2002; 1.

48. MOON TD, BRAWER MK, WILT TJ.: Prostate Intervention Versus Observation Trial (PIVOT): a randomized trial comparing radical prostatectomy with palliative expectant management for treatment of clinically localized prostate cancer. PIVOT Planning Committee. J Natl Cancer Inst Monogr 1995; 19: 69-71.

Dr. M. Arrizabalaga Moreno

Servicio de Urología

Hospital de Móstoles

C/ Río Júcar, s/n

28935 Móstoles (Madrid)

(Trabajo recibido el 11 junio de 2003) 